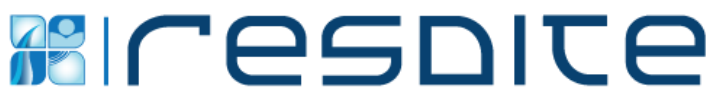

\section{Uso da Plataforma INDU em projeto de extensão na área}

\section{de Vigilância Alimentar e Nutricional}

Use of INDU platform in extension project in the area of Food and Nutrition Surveillance

\author{
Maira Pitta Farias', Emilly Marcela Mendes de Souza², Luana Salvador de Lemos ${ }^{3}$, \\ Davidson Alves dos Santos Lima ${ }^{4}$, Karolina de Cássia Lima da Silva Duarte ${ }^{5}$, \\ Magdala de Araújo Novaes ${ }^{6}$, Talita Helena Monteiro de Moura ${ }^{7}$
}

\section{Resumo}

Introdução: Os ambientes virtuais de aprendizagem constituem ferramentas de ensino capazes de ressignificar as práticas educativas, possibilitando a interação, 0 compartilhamento de saberes e a gestão do tempo. Objetivo: Demonstrar a importância do uso da plataforma INDU em um projeto de extensão na área de Vigilância Alimentar e Nutricional. Métodos: A extensão ocorreu em outubro de 2018 a abril de 2019 com a participação de 28 estudantes. Foi realizado o acompanhamento dos marcadores de consumo alimentar e nutricional com escolares menores de 10 anos e o registro na base de dados do SISVAN. Em paralelo às atividades de campo, os estudantes participaram de uma formação em ambiente virtual de aprendizagem e construíram um plano de intervenção, cujas ações visaram a promoção da alimentação adequada e saudável. Resultados: $O$ uso da plataforma INDU potencializou as ações desenvolvidas pelos extensionistas, pois, à medida em que ocorriam as atividades de campo, participaram de discussões no ambiente virtual de aprendizagem, suscitaram reflexões sobre a situação nutricional dos escolares e subsidiaram a construção de um plano de intervenção. Conclusão: A experiência possibilitou aos participantes o fazer saúde interdisciplinar, multiprofissional e a construção do pensamento crítico e inovador.

Palavras-Chave: Práticas Interdisciplinares. Obesidade. Educação em Saúde. Tecnologia Educacional.

\section{Abstract}

Introduction: Virtual learning environments are teaching tools capable of reframing educational practices, enabling interaction, knowledge sharing and time management. Objective: To demonstrate the importance of using the INDU platform in extension project in the area of Food and Nutrition Surveillance. Methods: The extension work took place from October 2018 to April 2019 with the participation of 28 students. Food and nutritional

\footnotetext{
1 Enfermeira. Universidade de Pernambuco. E-mail: mairapittafarias@gmail.com

2 Sanitarista. Universidade de Pernambuco. E-mail: emmsouza.sc@gmail.com

3 Sanitarista. Universidade de Pernambuco. E-mail: luanasalvador445@gmail.com

4 Graduando de Administração. Universidade de Pernambuco. E-mail: davidsonauniaeo@gmail.com

5 Mestra em Saúde Pública. Núcleo de Telessaúde do Hospital das Clínicas. Universidade Federal de Pernambuco. E-mail: karolina.silva@nutes.ufpe.br

6 Doutora em Bioinformatique. Núcleo de Telessaúde do Hospital das Clínicas. Universidade Federal de Pernambuco. E-mail: magdala.novaes@nutes.ufpe.br

7 Doutoranda em Educação Matemática e Tecnológica. Universidade Federal de Pernambuco. Secretaria de Saúde de Jaboatão dos Guararapes. E-mail: enf.talitamonteiro@gmail.com

Correspondência: Rua Professora Anunciada da Rocha Melo, 30, apt. 2102, Madalena, Recife, Pernambuco, 50710-390.
}

Rev. Saúde Digital Tec. Educ., Fortaleza, CE, v. 5, n. 1, p.124-136, jan./abr. 2020.

ISSN: 2525-9563 
consumption markers were monitored, with schoolchildren under 10 years old and registered in the SISVAN database. In parallel to the field activities, students participated in a training in virtual learning environment and built an intervention plan whose actions aimed at promoting adequate and healthy eating. Results: Education service integration initiatives contribute to the improvement of health indicators, changes in the work process in the various realities of the territory. Conclusion: The experience allowed participants to do interdisciplinary and multi-professional health, and the construction of critical and innovative thinking.

Keywords: Interdisciplinary Placement. Obesity. Health Education. Educational Technology.

\section{Introdução}

A modernidade trouxe consigo diversas mudanças ao mundo, dentre elas está o desenvolvimento da tecnologia, transformando nossa forma de viver, de ensinar e de aprender. Nas instituições de ensino, os ambientes virtuais de aprendizagem passaram a contribuir no processo ensino/aprendizagem, ressignificando as práticas educativas, percebendo o sujeito como autor/produtor ${ }^{1}$. A partir da utilização desses ambientes, estudantes e docentes podem experienciar atividades educativas que facilitam a interação, o estímulo, a motivação, a dedicação e a autogestão do tempo ${ }^{2}$.

A aplicação de diferentes métodos pedagógicos nas atividades virtuais e presenciais é conhecida como ensino híbrido ${ }^{3}$. A plataforma de Tele-educação INDU é um ambiente virtual baseado no sistema Moodle e criada no Núcleo de Telessaúde do Hospital das Clínicas da Universidade Federal de Pernambuco (NUTES-HC-UFPE). Ela tem o intuito de "promover a educação e disseminar o conhecimento na área de saúde e tecnologias associadas"4. Neste ambiente virtual, é possível realizar fóruns, cursos, palestras on-line, comunidades de práticas, segunda opinião formativa e outras atividades que potencializam as ações de educação permanente em saúde. Além disso, existe a possibilidade de desenvolver projetos que envolvam estudantes e profissionais em serviço, fortalecendo assim as ações de integração, ensino e serviço. Estas vêm se construindo paulatinamente por meio de experiências exitosas, que proporcionaram contribuições significativas tanto para a academia quanto para os profissionais de saúde e a comunidade. Mesmo diante dos desafios, a formação em serviço potencializa o aprendizado em cenários reais de atenção à saúde. As vivências no contexto interligado entre ensino, serviço e comunidade possibilitaram mudanças, com vistas a fortalecer as Políticas de Saúde ${ }^{5}$.

A "Política Nacional de Alimentação e Nutrição" (PNAN) contempla a diretriz "Vigilância Alimentar e Nutricional", no planejamento da atenção nutricional no 
SUS, de modo que uma de suas finalidades é a promoção da alimentação adequada e saudável no âmbito individual e coletivo ${ }^{6,7}$. O programa de prevenção, controle e tratamento da obesidade infantil, "Crescer Saudável", foi estruturado no intuito de desenvolver ações articuladas a serem implementadas na Rede de Atenção à Saúde do SUS para garantir o adequado acompanhamento do crescimento e desenvolvimento na infância. Assim, visa prevenir, controlar e tratar a obesidade infantil junto à população no âmbito do Programa Saúde na Escola (PSE), contribuindo com a prevenção, o controle e o tratamento da obesidade infantil ${ }^{8}$.

No Brasil, entre 2006 a 2018, a variação temporal de indicadores do Vigitel (Vigilância de Fatores de Risco e Proteção para Doenças Crônicas por Inquérito Telefônico), na população adulta das 26 capitais e do Distrito Federal, mostraram que a obesidade, o excesso de peso e a diabetes evoluíram de forma desfavorável e significativa". De acordo com a "Pesquisa Nacional de Saúde do Escolar" de 2015, o indicador de excesso de peso apresentou prevalência, cerca de $23,7 \%$, o que corresponde ao total estimado de 3 milhões de escolares. Entre os dois sexos houve pouca variação. No total da população-alvo, 8,3\% dos meninos e 7,3\% das meninas foram considerados obesos ${ }^{10}$.

Ao observar a importância dos cuidados em saúde na infância para formação de adultos saudáveis e para redução da mortalidade infantil, o Ministério da Saúde criou a Política Nacional de Atenção Integral à Saúde da Criança (PNAISC) que coloca em um dos seus eixos estratégicos 0 aleitamento materno e a alimentação complementar saudável ${ }^{11}$.

No município de Jaboatão dos Guararapes, pertencente ao estado de Pernambuco, o programa "Crescer Saudável", além de ter se desenvolvido em seu caráter originalmente intersetorial com a participação de gestores, profissionais da atenção básica e educação, também contou com a participação de 28 estudantes que desenvolveram atividades de campo pelo Programa Saúde na Escola (PSE) e vinculadas a um projeto de extensão. Os estudantes foram inseridos em uma formação diretamente relacionada ao conteúdo do programa "Crescer Saudável" e planejaram uma intervenção com base nos dados do estado nutricional e do consumo alimentar dos escolares.

A extensão universitária teve como objetivo fazer uma ponte entre a universidade e a população, estendendo o conhecimento produzido na academia à sociedade. Uma de suas características é a horizontalidade e o conhecimento da cultura local onde se desenvolve, assumindo um compromisso promover mudanças $^{12}$. Desta forma, a extensão universitária contribui significativamente para formação em saúde, estando atrelada 
à articulação entre gestão, atenção, ensino e controle/participação social ${ }^{13}$.

Diante do exposto, o presente relato objetiva demonstrar a importância do uso da plataforma INDU em projeto de extensão na área de vigilância alimentar e nutricional. A troca de saberes entre gestores, estudantes, profissionais da saúde e da educação, suscitaram reflexões sobre elementos da realidade do território e subsidiaram o planejamento das ações de promoção da alimentação saudável e do combate à obesidade infantil nas unidades de saúde participantes.

\section{Métodos}

Este artigo envolve um estudo descritivo sobre o projeto de extensão "Crescer Saudável" desenvolvido no em Jaboatão dos Guararapes. O projeto surgiu como forma de atender às exigências do programa de prevenção, controle e tratamento da obesidade infantil "Crescer Saudável" do Ministério da Saúde. O estudo foi realizado de outubro de 2018 a abril de 2019. Participaram 24 estudantes dos cursos de Enfermagem e Nutrição de universidades públicas e privadas das cidades de Recife e Jaboatão dos Guararapes e quatro residentes do Programa de Residência Multiprofissional em Atenção Básica e Saúde da Família da Secretaria de Saúde do Jaboatão dos Guararapes.

$\mathrm{Na}$ primeira etapa do projeto foram planejadas ações em escolas municipais, já estavam vinculadas ao Programa Saúde na Escola (PSE), para o preenchimento de uma ficha com marcadores de consumo alimentar e outra para o cadastro de acompanhamento nutricional do sistema ESUS e/ou SISVAN.

O município do Jaboatão dos Guararapes está estruturado territorialmente em sete regionais de saúde. Foram realizadas entrevistas com 5.191 escolares menores de 10 anos, em 16 escolas municipais, especificamente nas Regionais I, II, III, IV, VI e VII. Posteriormente, ocorreram as atividades nessas mesmas escolas.

Para realização das ações foi feita articulação entre a Secretaria de Saúde e Secretaria de Educação do município a partir do Programa Saúde na Escola (PSE). Durante o projeto, os estudantes contaram com a supervisão e o apoio das equipes de saúde da família, gestores da Secretaria Municipal de Saúde e profissionais das escolas.

A segunda etapa do projeto consistiu na digitação da ficha de cadastro e acompanhamento nutricional no banco de dados do Sistema de Vigilância Alimentar e Nutricional (SISVAN) do Ministério da Saúde. Para isso, foi utilizado o instrumento "Marcadores de Consumo Alimentar", que proporcionou a obtenção de dados relacionados à saúde nutricional dos escolares.

Concomitante à coleta dos dados nas escolas e à digitação na Secretaria de 
Saúde de Jaboatão dos Guararapes, foi utilizada a Plataforma INDU da Rede NUTES-UFPE para realização de atividades em ambiente virtual de aprendizagem. O objetivo era promover trocas de experiências para a elaboração do plano de intervenção nas escolas, construído a partir da interpretação dos dados coletados nas fichas e das vivências em campo. O ambiente virtual contou com fóruns de discussão, materiais para estudo (artigos e publicações do Ministério da Saúde) e uso da ferramenta Wiki® para elaborar o plano de intervenção.

\section{Resultados}

O projeto envolveu equipes da Atenção Básica e da Educação Permanente da Secretaria da Saúde de Jaboatão dos Guararapes com o objetivo de realizar o preenchimento das fichas de cadastramento e acompanhamento nutricional de crianças, no Sistema de Vigilância Alimentar e Nutricional (SISVAM), que envolvem os marcadores de consumo alimentar e a verificação do peso e da altura.

Inicialmente, foi realizada a inscrição dos extensionistas (estudantes de graduação e pós-graduação na modalidade residência), por meio de formulário on-line Google Forms. Posteriormente, foi realizada a convocação dos estudantes para participarem das oficinas formativas com a apresentação do projeto, da temática central, dos materiais de coleta, bem como, promovendo a troca de experiências.

Já no campo, a primeira etapa das atividades envolveu estabelecer o vínculo entre estudantes universitários e os residentes dos cursos de Enfermagem, Nutrição, Educação Física, Fonoaudiologia juntamente com os profissionais das Unidades de Saúde da Família (USFs) e das escolas. As ações nas escolas tinham inicialmente o objetivo de coletar os dados da situação nutricional por meio do preenchimento das fichas. Porém, em virtude da articulação positiva de algumas equipes de saúde da família com os extensionistas, foi possível ir além, desenvolvendo ações de educação em saúde como o teatro de fantoches e as rodas de conversas com os escolares. Estas atividades foram realizadas após a coleta de dados para não interferir nos resultados, visto que, mediante 0 preenchimento das fichas de cadastramento e de acompanhamento nutricional, os estudantes obtiveram informações sobre os hábitos alimentares dos escolares.

Nestes momentos, destacou-se a participação dos familiares e dos cuidadores dos escolares, principalmente as mães, que se aproximaram dos espaços, na busca de informações sobre a alimentação saudável.

$\mathrm{Na}$ segunda etapa, foi realizada a digitação das fichas de cadastramento e de acompanhamento nutricional com 
marcadores de consumo alimentar na plataforma do SISVAN. Neste momento, os extensionistas enfrentaram dificuldades relacionadas às instabilidades do SISVAN. Contudo, os cadastros foram concluídos com sucesso, totalizando 5.191 crianças, na faixa etária de dois a 10 anos.

A terceira etapa aconteceu concomitante à digitação, no Ambiente Virtual de Aprendizagem (Plataforma INDU) do Núcleo de Telessaúde de Pernambuco (RedeNUTES). O uso dessa plataforma faz parte de uma cooperação técnica firmada entre o NUTES/UFPE e a Coordenação da Educação Permanente da Secretaria Municipal de Saúde do Jaboatão dos Guararapes. A plataforma é de acesso restrito e privativo. Portanto, foi necessário que cada extensionista e demais colaboradores realizassem cadastro para acessar. As atividades no ambiente virtual foram construídas por professores e colaboradores. Na página inicial, havia um texto de "boas vindas" com um breve resumo sobre o projeto e sobre o desenvolvimento das atividades na plataforma. Em seguida, era possível assistir um vídeo intitulado "Webpalestra Programa Crescer Saudável", disponível na plataforma YouTube e produzido pelo Telessaúde.

A plataforma on-line tinha o objetivo de oferecer material administrativo, específico deste projeto de extensão, além de arquivos com conteúdo relacionado à Política de Alimentação e Nutrição. Os fóruns de discussão foram disponibilizados semanalmente, fomentando as reflexões sobre alimentação saudável e situações vivenciadas na coleta de dados nas escolas. Entre os materiais administrativos, um dos mais relevantes para o funcionamento da extensão foi o "Manual do extensionista", o qual definia as etapas para o planejamento e o andamento das ações do projeto.

Semanalmente, era postado na plataforma um fórum com uma determinada atividade para as trocas de experiências e de conteúdo formativo. No ambiente virtual, os extensionistas foram distribuídos em dois grupos, sendo uma das primeiras atividades a escolha do nome de cada grupo, que foram denominados respectivamente "Divertidamente Saudável" e "+ Criança". Durante as postagens, foram discutidas e compartilhadas as vivências em campo, na medida em que ocorriam as visitas para coleta de dados nas escolas.

Cada grupo construiu um plano de intervenção, considerando os dados da situação nutricional dos escolares e vivências nas escolas. $O$ plano de intervenção e os dados consolidados foram apresentados no I Seminário do Projeto de Extensão "Crescer Saudável" que contou com a participação de representantes da gestão de saúde e de educação do município, estudantes, extensionistas e profissionais das equipes de Saúde da Família. Durante o evento, os 
extensionistas apresentaram a experiência neste projeto e entregaram o plano de intervenção a um representante de cada regional de saúde, a fim de estimular a continuidade das ações nas escolas. 0 evento teve a participação de todos os atores envolvidos no projeto e marcou a finalização das atividades da extensão.

\section{Discussão}

Durante o caminhar da extensão foram observadas algumas dificuldades, entre elas, o desafio do estudante conciliar a atividade extracurricular com as atividades curriculares. A proposta da extensão "Crescer Saudável" foi concebida com objetivo de integrar diferentes metodologias de ensino - aprendizagem, nos ambientes presencial e on-line. Silva (2017) enfatiza que o ensino híbrido possibilita aos estudantes aprendizado no seu próprio ritmo de forma criativa e contemporânea ${ }^{14}$.

O uso da plataforma INDU foi fundamental para apoiar os extensionistas nas atividades formativas, relacionadas ao projeto. Também possibilitou os diálogos entre os participantes das regionais de saúde, mesmo estando distantes territorialmente, em virtude da magnitude territorial do município de Jaboatão. Portanto, a carga horária da extensão, envolveu atividades em campo, reuniões presenciais e atividades on-line (digitação das fichas, participação dos fóruns e construção do plano de intervenção). $\mathrm{Na}$ plataforma era postado semanalmente uma atividade reflexiva/educativa, relacionada a vigilância alimentar e nutricional, onde era proposto para o extensionista a leitura de materiais, vídeos/documentários e compartilhamento de experiências. $O$ desenvolvimento das atividades da extensão na plataforma online e nos encontros presenciais possibilitou a participação efetiva dos extensionistas, visto que havia dificuldade em cumprir a carga horária totalmente presencial.

Como já era esperado, alguns extensionistas tiveram dificuldade para realizar as atividades on-line por nunca terem estudado em ambiente virtual de aprendizagem ou por estarem "acostumados" (relato de extensionista) com atividades on-line desenvolvidas exclusivamente no formato de videoaulas e questionários. O que caracteriza um ambiente com pouca interação. Neste projeto, foram construídas atividades que estimulavam a criatividade. Campos e Santos (2016) abordam em estudo analisando sobre às tecnologias de informação na Educação Permanente em Saúde, que estudantes adaptados aos métodos convencionais de aprendizado tendem à apresentar reflexões acerca dos novos recursos de ensino ${ }^{15}$.

Outro aspecto importante desta experiência, consistiu nas ações desenvolvidas nas escolas. Um dos propósitos da extensão universitária é 
alcançar um retorno social, e sobre isso, além dos materiais produzidos durante a extensão e o preenchimento das fichas de marcadores alimentar, algumas escolas disponibilizaram estrutura mínima de espaço e horário no cronograma sem prejuízo escolar, para realizar as ações educativas após o preenchimento das fichas, onde as crianças e muitos familiares demonstraram interesse

e responsabilidade com as novas informações que tiveram acesso. Segundo Gomes, Dallas e Rosso (2019) ${ }^{16}$.

[..] A extensão universitária tem por finalidade promover mudança no método docente e aprendizagem compartilhados e interdependentes entre sociedade e universidade.

As dificuldades deste estudo envolveram inicialmente, a pouca articulação entre os extensionistas de diferentes cursos e instituições inseridos no mesmo território municipal, principalmente devido as barreiras territoriais. Mas, paulatinamente, os diálogos se fortaleceram por meio da plataforma INDU, efetivando a troca de saberes e subsidiaram o planejamento das ações de promoção da alimentação saudável e do combate à obesidade infantil nas unidades de saúde participantes.

\section{Conclusão}

A proposta de um projeto de extensão é ultrapassar os muros das
Universidades e associar a teoria com a prática. No "Crescer Saudável", as ações foram desenvolvidas de forma multidisciplinar, envolvendo diferentes setores da Saúde (Educação Permanente, Políticas Estratégicas e Atenção), promovendo a integração entre acadêmicos e comunidade para obter resultados quantitativos e qualitativos. Portanto, o desenvolvimento desta formação na modalidade híbrida potencializou o pensamento críticoreflexivo sobre alimentação, educação, saúde e sociedade através do compartilhamento e da discussão por meio dos fóruns realizados na plataforma INDU, bem como nos encontros presenciais com objetivo formativo.

Além de ser um espaço público privilegiado para construção de cidadania e de mobilização popular, a escola também é lugar de convivência social que permite o desenvolvimento de relações favoráveis à promoção da saúde através da compreensão e da superação de seus determinantes sociais, considerando ainda os aspectos da intersetorialidade.

O projeto de extensão "Crescer Saudável" pôde então alcançar diversos atores que se empoderaram com as temáticas de alimentação saudável e de promoção da saúde, concretizando uma experiência de aprendizagem significativa aos estudantes e aos demais colaboradores. 
Portanto, percebeu-se a importância do papel e da responsabilidade de cada membro da equipe da Estratégia Saúde da Família, academia e comunidade, mostrando que a sinergia do trabalho integrado é capaz de fomentar a promoção da saúde.

\section{Agradecimentos}

À Secretaria de Saúde de Jaboatão dos Guararapes pela sensibilidade na percepção dos altos índices de agravos decorrentes do estilo de vida na população jaboatonense e no enfrentamento desses por meio da intersetorialidade.

Aos profissionais das equipes de Saúde da Família do município que desenvolvem diariamente suas funções próximo a população, em prol da saúde individual e coletiva.

Aos estudantes, extensionistas e residentes, que viabilizaram e desenvolveram as atividades do projeto, colocando-se enquanto verdadeiros atores sociais no decorrer da extensão.

Por último, à rede NUTES/UFPE pela parceria e colaboração.

\section{Referências}

1. Maciel IM. Educação à distância.

Ambiente virtual: construindo significados. Bol Téc. do SENAC. 2002; 28(3): 38-45.

2. Holanda VR de, Pinheiro AKB, Pagliuca LMF. Aprendizagem na educação online: análise de conceito. Rev Bras Enferm [Internet]. 2013 [acesso em 2019 Dec 01]; 66(3): 406-11. Disponível em: http://www.scielo.br/scielo.php?script=sci arttext\&pid=S0034-

$71672013000300016 \& \operatorname{lng}=p t \& t \operatorname{lng}=p t$

3. Almeida Júnior J. Ensino híbrido: personalização e tecnologia na educação. Rev Thema [Internet]. 2017 [acesso em 2019 Nov 10]; 14(2): 336-40. Disponível em:

http://revistathema.ifsul.edu.br/index.php/t hema/article/view/429

4. Núcleo de Telessaúde do Hospital das Clínicas da Universidade Federal de Pernambuco. INDU [homepage na internet]. Recife: UFPE; 2019. [atualizada em 2019 Dec 10; acesso em 2019 Oct 13]. Disponível em:

http://www.nutes.ufpe.br/indu/course/index .php?categoryid $=4$

5. Kuabara CT de M, Sales PR de S, Marin MJS, Tonhom SF da R. Integração ensino e serviços de saúde: uma revisão integrativa da literatura. Reme Rev Min Enferm [Internet]. 2014 [acesso em 2019 Dec 08]; 18(1): 195-201. Disponível em: http://www.gnresearch.org/doi/10.5935/14 15-2762.20140015

6. Ministério da Saúde (BR). Marco de Referência da Vigilância Alimentar e Nutricional na Atenção Básica. Brasília: Ministério da Saúde; 2015. 7.

7. Ministério da Saúde (BR). Política Nacional de Alimentação e Nutrição. Brasília: Ministério da Saúde; 2013. 8. Ministério da Saúde (BR). Começa o período de adesão ao Crescer Saudável 2019-2020

[homepage na internet]. Brasília: Ministério da Saúde; 2019. [atualizada em 2019 Apr 05; acesso em 2019 Oct 10].Disponível em: https://aps.saude.gov.br/noticia/5394 9. Ministério da Saúde (BR). Vigitel Brasil 2018: vigilância de fatores de risco e proteção para doenças crônicas por inquérito telefônico. Brasília: Ministério da Saúde; 2019.

10.Instituto Brasileiro de Geografia e Estatística. Pesquisa nacional de saúde do escolar 2015. Rio de Janeiro: IBGE; 2016.

11. Ministério da Saúde (BR). Política Nacional de Atenção Integral à Saúde da Criança: orientações para implementação [Internet]. Brasília: Ministério da Saúde; 
2018 [acesso em 2019 Nov 10].

Disponível em:

http://www.saude.pr.gov.br/arquivos/File/P

olitica_Nacional_de_Atencao_Integral_a_

Saude_da_Crianca_PNAISC.pdf

12.Nogueira MDP, editor. Avaliação da

Extensão Universitária: práticas e discussões da Comissão Permanente de

Avaliação da Extensão. Belo Horizonte:

FORPROEX/CPAE; PROEX/UFMG; 2013.

13.Ceccim RB, Feuerwerker LCM. O

Quadrilátero da Formação para a Área da

Saúde: Ensino, Gestão, Atenção e

Controle Social. Rev Saúde Coletiva

[Internet]. 2004 [acesso em 2019 Dec 01];

14(1): 41-65. Disponível em:

http://www.scielo.br/scielo.php?pid=S0103

$73312004000100004 \&$ script=sci_abstract

\&tlng=pt.

14. Silva JB da. O contributo das

tecnologias digitais para o ensino híbrido:

O rompimento das fronteiras espaço-

temporais historicamente estabelecidas e

suas implicações no ensino. Rev Estud

em Linguagens e Tecnol [Internet]. 2017

[acesso em 2019 Dec 01]; 15 (2): 11.

Disponível em:

http://www.artefactum.rafrom.com.br/index

.php/artefactum/article/view/1531

15. Campos KA, Santos FM dos. A

educação a distância no âmbito da

educação permanente em saúde do

Sistema Único de Saúde (SUS). Rev do

Serviço Público [Internet]. 2016 [acesso

em 2019 Dec 01]; 67 (4): 603. Disponível

em:

https://revista.enap.gov.br/index.php/RSP/

article/view/105516.

16.Gomez SEM, Corte MGD, Rosso GP.

A Reforma de Córdoba e a educação

superior. Rev Inter de Educ Sup [Internet].

2019 [acesso em 2019 Dec 01]; 5: 1-21.

Disponível em:

https://periodicos.sbu.unicamp.br/ojs/index

.php/riesup/article/view/8653655/19027 


\section{Anexos}

Figura 1: Tela inicial do ambiente virtual do crescer saudável no INDU

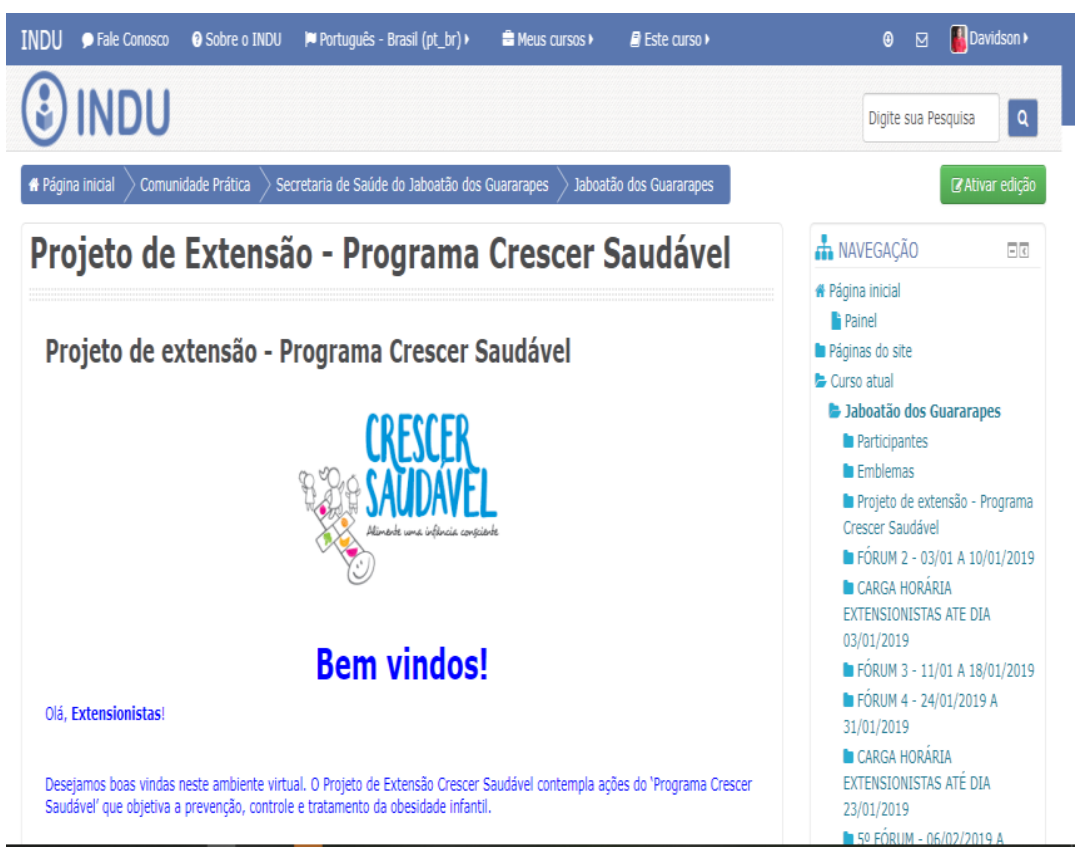

Fonte: http://www.nutes.ufpe.br/indu/course/view.php?id=788. Acesso em: 13/10/2019. 
Figura 2: Fóruns de discussão e construção das atividades no INDU

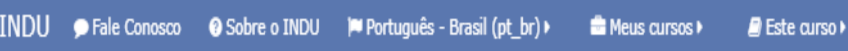

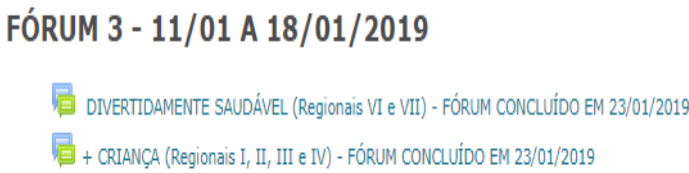

FÓRUM 4 - 24/01/2019 A 31/01/2019

曷 DIVERTIDAMENTE SAUDÁVEL (Regionais VI e VII) - FóRUM CONCLUídO EM 06/02/2018

酋 + CRIANÇA (Regionais I, II, III e IV) - FÓRUM CONCLUíDO EM 06/02/2018

局 Atividade Lúdica! (Todas as regionais) - FÓRUM CONCLUído EM 06/02/2018

\section{CARGA HORÁRIA EXTENSIONISTAS ATÉ DIA 23/01/2019}

'E. CARGA HORÁRIA EXTENSIONISTAS ATÉ DIA 23/01/2019

50 FÓRUM - 06/02/2019 A 13/02/2019

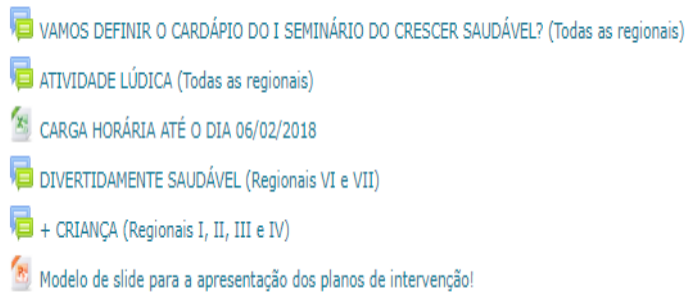

Fonte: http://www.nutes.ufpe.br/indu/course/view.php?id=788. Acesso em: 13/10/2019. 


\section{Como citar este artigo}

Farias MP, Souza EMM de, Lemos LS de, Lima DAS, Duarte KCLS, Novaes MA, Moura THM de. Uso da Plataforma INDU em projeto de extensão na área de Vigilância Alimentar e Nutricional. Revista de Saúde Digital e Tecnologias Educacionais. [online], volume 5, n. 1. Editor responsável: Luiz Roberto de Oliveira. Fortaleza, mês e ano, p. 124-136. Disponível em: http://periodicos.ufc.br/resdite/index. Acesso em "dia/mês/ano".

Data de recebimento do artigo: 15/10/2019

Data de aprovação do artigo: 12/02/2020

Data de publicação: 17/04/2020 